\title{
Effect of leg wrapping technique on prevention of spinal induced hypotension and fetal acidosis during cesarean section: Randomized controlled trial
}

\author{
Samah Nasser Abd El-Aziz El-Shora, Amina Mohamed Rashad El-Nemer \\ Woman's Health and Midwifery Nursing Dep. Faculty of Nursing, Mansoura University, Egypt
}

Received: August 26, 2019

DOI: $10.5430 /$ jnep.v10n2p70
Accepted: October 23, 2019

Online Published: November 13, 2019

URL: https://doi.org/10.5430/jnep.v10n2p70

\begin{abstract}
Background and aim: Hypotension during cesarean section (CS) under spinal anesthesia has been a subject of scientific study for more than 50 years and the search for the most effective strategy to achieve hemodynamic stability remains challenging. Aim: The study was carried out to apply leg wrapping technique for the prevention of spinal-induced hypotension (SIH) during CS. Methods: Randomized Controlled Trial design was utilized at cesarean delivery operating room Mansoura General Hospital in El-Mansoura City during the period from May 2018 to November 2018. A purposive sample of 88 pregnant women, assigned randomly to an intervention group $(n=44)$ in which their legs wrapped with elastic crepe bandage and control group $(n=44)$ in which no wrapping was done. Data collected for maternal, neonatal hemodynamic and signs of hypotension, the feasibility of application and cost analysis.

Results: There was a statistically significant difference in the incidence of SIH and Ephedrine use among both groups (18.20\% in leg wrapping group whereas $75 \%$ in control group). In addition, neonatal acidosis and NICU admission were less among leg wrapping group $(11.40 \%, 9.10 \%$ respectively). Economically, leg wrapping technique was cost effective compared to the cost of the hospital regimen for treating SIH and admission to (NICU).

Conclusion and recommendations: Leg wrapping technique was cost effective and an efficient method for decreasing SIH, neonatal acidosis and Ephedrine administration. It is recommended to apply leg wrapping technique in maternal hospitals' protocol of care for decreasing SIH during CS.
\end{abstract}

Key Words: Leg wrapping, Spinal induced hypotension, Cesarean Section

\section{INTRODUCTION}

Cesarean birth has become the most common hospital-based operative procedure. World Health Organization (WHO) reported that the rate of CS has been raised from 5\%-25\% during the last 20 years at the national and international levels. For example, in Egypt, the cesarean delivery rate has raised dramatically from $27.6 \%$ in 2010 to $52 \%$ in 2014 and 65.5\% in Dakhalia in 2014. According to the anecdotal re- port, the rate of cesarean delivery was $65.44 \%$ at Mansoura General Hospital in 2017, 61.29\% in 2018 and $65.80 \%$ in mid-year of 2019. ${ }^{[1,2]}$

The choice of anesthesia for a cesarean birth is achieved by balancing women's preferences with the hazards and benefits of a specific procedure to the woman and her baby. ${ }^{[3]}$ It has equally increasingly shifted from general to spinal anesthesia. It is quite easy to perform, has lower risks, reliable, safe,

\footnotetext{
*Correspondence: Samah Nasser Abd El-Aziz El-Shora; Email: dr_samah1987@yahoo.com; Address: Woman’s Health and Midwifery Nursing Dep. Faculty of Nursing, Mansoura University, Egypt.
} 
avoids the depressant effects of anesthetic drugs, allowing the woman to be awake during and immediately after surgery and provides excellent operating conditions for CS compared to general anesthesia. ${ }^{[4]}$

Due to the invasive nature of spinal anesthesia, it is still associated with considerable side effects, of which hypotension is the most frequent with the incidence of $70 \%-80 \%$. In the pregnant women, spinal-induced hypotension along with the inferior vena cava compression via the gravid uterus additional inhibits venous return, and if unmanaged, this process may produce maternal hypotension and uterine hypoperfusion. ${ }^{[5]}$

Spinal-induced hypotension is the most common clinical issue of spinal anesthesia during CS. It is frequently accompanied by maternal and neonatal risks. Without intervention, the incidence of SIH during CS is estimated to be as high as $85 \%$. So, prevention of hypotension and its related negative effects under spinal anesthesia for CS has been the "holy grail" of obstetric anesthesia for decades. ${ }^{[6,7]}$

Preventing spinal hypotension in cesarean delivery has been one of the main research targets within obstetric anesthesia. A stable hemodynamic condition improves maternal safety and reduces morbidity in the form of nausea, distorted consciousness, and fetal acidosis. ${ }^{[8]}$ Many methods to decrease the risk of hypotension have been studied, which involve ensuring appropriate maternal position with uterus away from vena cava, fluids infusion to rise blood volume and administration of ephedrine and phenylephrine to increase vascular tone. ${ }^{[3,9,10]}$

Leg wrapping is helpful in improving venous return and therefore improve blood pressure. Wrapping of lower limbs during CS is an evidence-based nursing procedure, however, it is not applied in the Egyptian health care system. Wrapping of lower limbs with an elastic bandage is an easy, noninvasive and non-pharmacological technique that furthermore may be utilized during less sophisticated and economical settings, such as in developing countries for preventing spinal-induced hypotension and its subsequent adverse effect on the woman as well as on the newborn.

The degree of effectiveness of various preventive methods is controversial and there is no established ideal technique. Hypotension has not to be underestimated since scientific data suggested that it may be accompanied by neonatal acidosis and low Apgar scores. There is a lack of studies on neonatal outcome. Therefore, this study investigated the effect of leg wrapping on the neonatal outcome. ${ }^{[11]}$

Published by Sciedu Press

\subsection{Aim of the study}

The study aimed to apply the leg wrapping technique for prevention of spinal-induced hypotension during CS.

\subsection{Study hypotheses}

To achieve the present study aim, two hypotheses were tested:

Hypothesis I: Application of leg wrapping technique during cesarean section will prevent spinal-induced hypotension.

Hypothesis II: Application of leg wrapping technique during cesarean section will prevent neonatal risk for acidosis.

\section{SUBJECTS AND METHOD}

\subsection{Design}

Randomized Controlled Trial (RCT) design was utilized in this study.

\subsection{Setting}

The current study was carried out at the cesarean delivery operating room Mansoura General Hospital that delivers a broad series of health services at Delta Region. Obstetrical and gynecological department occupied with 14 beds and operating room consists of 3 rooms, each room occupied with one operating table. About 40 cesarean deliveries are performed monthly. The flow rate of cesarean deliveries was 536 cases in 2017, 597 cases in 2018 and 306 cases in mid-year of 2019.

\subsection{Sampling}

A purposive sample of 88 pregnant women was recruited. The inclusion criteria are as follows: normal pregnancy, full term (37-41 weeks of gestation), singleton pregnancy, age ranged from 19 to 35 years old, admitted to the CS operating room and agreed to give informed consent to participate in the study. Exclusion criteria include the following: a history of deep venous thrombosis of their legs, superficial thrombophlebitis of their lower extremities, morbid obese women (greater than $110 \mathrm{~kg}$ ). Pregnant women who fulfilled the inclusion criteria were assigned to one of two groups. Intervention group (leg wrapping group) $(n=44)$ who received conventional preoperative care of cesarean section, in addition to the leg wrapping before spinal anesthesia. Control group $(n=44)$ who received the traditional preoperative care of cesarean section.

\subsection{Sample size}

A previous study showed that $40.1 \%$ of women with leg wrapping had hypotension compared to $70.6 \%$ in women without leg wrapping. ${ }^{[12]}$ Considering a level of significance $=5 \%$, power $=80 \%$, with a test type is two-sided, the formula for calculating size of the sample is $n=$ $\left.\left[2\left(Z_{\alpha / 2}+Z_{\beta}\right)\right]^{2} \times p(1-p)\right] /(p 1-p 2)^{2}$. 
Based on the above formula, size of the sample needed for each group is 44 .

\subsection{Method of data collection}

Data were collected using one tool, it included 6 parts:

Part (1): It covered the participants' sociodemographic data such as age, education, residence, weight, height, etc.

Part (2): It covered the participant's obstetric history such as gravidity, parity, duration of pregnancy, etc.

Part (3): Maternal hemodynamic and signs of hypotension assessment: It was developed by the researcher after reviewing related literature ${ }^{[12-15]}$ to assess maternal hemodynamic data and signs of hypotension such as nausea and vomiting. Part (4): Neonatal assessment sheet: To record the umbilical arterial blood PH, and Apgar score at first and fifth minutes. Part (5): Feasibility of the application questionnaire: It was developed by the researcher after reviewing related literature; it included two open questions representative of (1) Positive factors for applying leg wrapping technique during cesarean section. (2) Barriers of applying leg wrapping technique from researcher and health care providers point of views.
Part (6): Cost analysis: To assess the cost of applying leg wrapping technique by calculating the cost of the technique against the cost of hospital regimen for treating hypotension and admission to the Neonatal Intensive Care Unit (NICU).

\subsection{The validity of the tool}

Content validity of the study tool was confirmed by three experts in the specialty of the study; one in obstetric medicine field and two in maternity nursing. Based on the expert's suggestions, minor modifications were done.

\subsection{Reliability of the tool}

The test for reliability and internal consistency for the questionnaire showed Cronbach's alpha value $(\alpha=0.91)$ and hence the questionnaire showed high reliability.

\subsection{Pilot study}

The pilot study was carried out on $10 \%$ of the sample size (9 pregnant women with the pre-assigned criteria), to test the practicability of the intervention. The women involved in the pilot study were excluded from the analyzed sample. Pilot study phase was conducted for one month (May 2018).

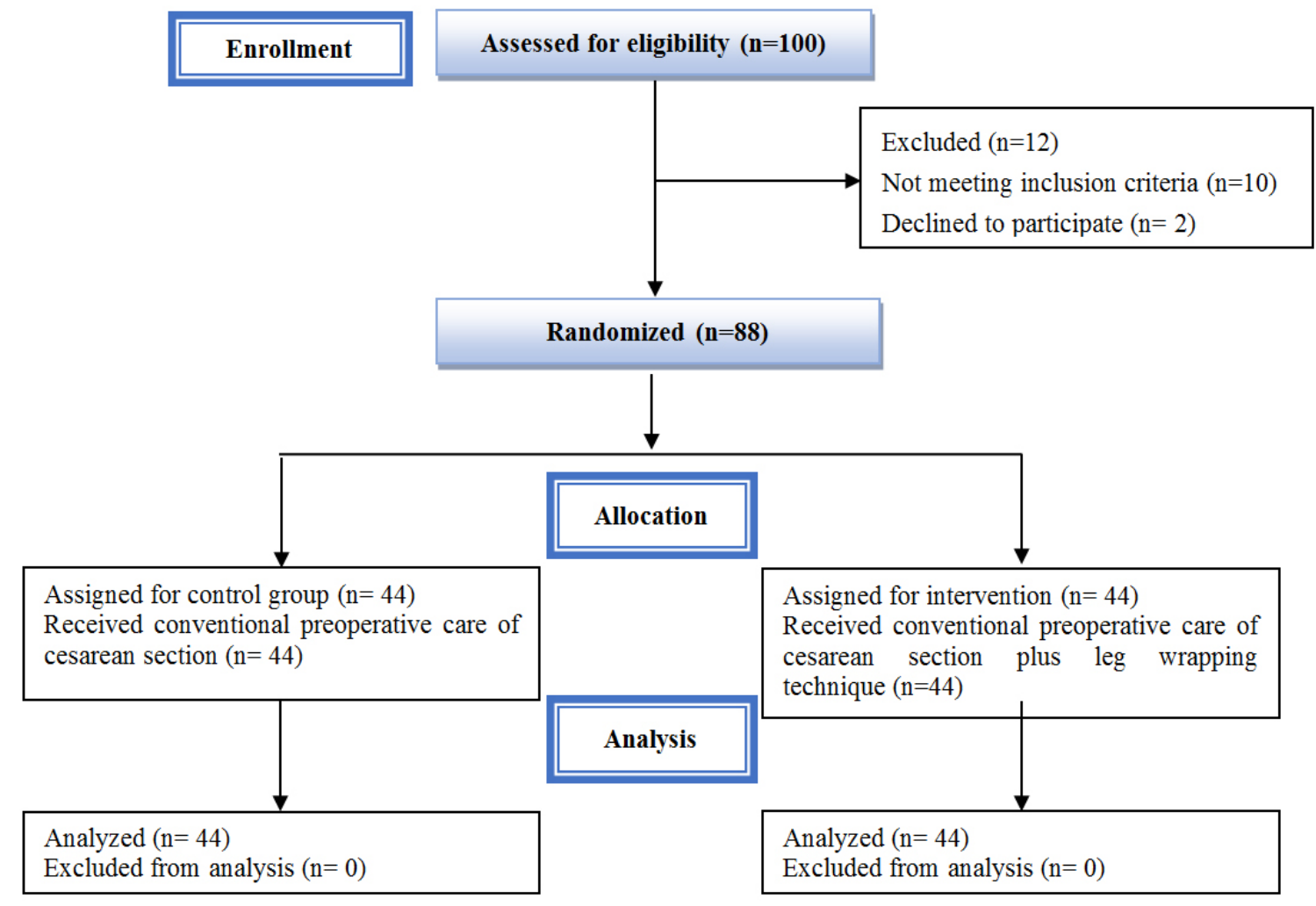

Figure 1. Flowchart of the sample study

\subsection{Ethical considerations}

Ethical approval was attained from the Research Ethics Committee at the Faculty of Nursing - Mansoura University. Writ- ten consent was attained from each woman following explaining the aim of the study. Involvement in the study was voluntary and each woman had the right to withdraw from 
the study at any time with no consequences. Confidentiality of the collected data was conserved and the results used as a component of the essential research for the doctoral study, in addition to for future publications and education.

\subsection{Data collection}

The actual field work of the study occurred for 6 months period, starting in May 2018 \& finished in November 2018. The data were collected three days per week from 9 a.m. to 7 p.m. On arrival at the hospital, all potential participants had received their preoperative care according to the set protocol of care; specifically pregnant women were nil per oral for $8 \mathrm{hrs}$. before surgery and canulated in the left antecubital vein with $16 \mathrm{G}$ cannula with a continuing Ringer's lactate infusion and observed by non-invasive blood pressure and pulse oximeter.

Thereafter, the researcher provided a clear explanation about the study nature to each eligible pregnant woman and getting the written informed consent from every woman accepted to share in the study. Each one was allotted either to the leg wrapping group or to the control group. Groups' assigned based on Systematic Random technique, where the Odd numbers of eligible women were allocated to the leg wrapping group, while the Even numbers of eligible women were allocated to the control group (Flowchart of the study sample shown in Figure 1).

At the baseline assessment, the researcher explained the study tool to each woman, asked the woman and recorded her answers in the data collection sheet. Then started to take baseline values of heart rate and blood pressure as the average of 3 consecutive readings 5 minutes interval before administering spinal anesthesia.

For the intervention group, lower limbs were raised to $45^{\circ}$ and wrapped from ankle to above the knee by two elastic bandages ( $15 \mathrm{~cm}$ width, $3 \mathrm{~m}$ stretched length). The bandages were wrapped tight enough that the women felt the tightness, yet it was comfortable and not painful. Leg wrapping technique was applied until the end of the cesarean delivery and was taking off before the woman's discharge from the operating room.

The spinal anesthesia was performed with $2.5 \mathrm{ml}$ of $0.5 \%$ hyperbaric Bupivacaine through an $18 \mathrm{G}$ needle in the interspace $\mathrm{L} 2 / \mathrm{L} 3$ or $\mathrm{L} 3 / \mathrm{L} 4$ while the woman in a sitting position under aseptic technique. After spinal anesthesia, the control group was immediately placed supine while the intervention group placed supine with $15^{\circ}$ left lateral tilt. $15^{\circ}$ Left lateral tilt attained by keeping a wedge under the right buttock.

Published by Sciedu Press
For both groups (the intervention and control groups) the following parameters were recorded intraoperatively for the study including: heart rate, systolic blood pressure, diastolic blood pressure and mean arterial pressure every five min. following spinal anesthesia till the end of surgery, nausea and vomiting.

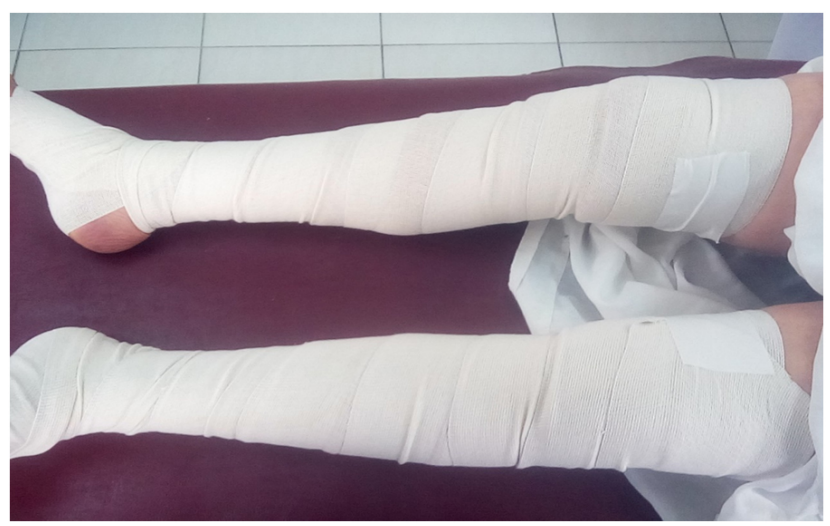

Figure 2. Using Leg Wrapping Description: Samah Nasser Abd El-Aziz El-Shora Documentation

Hypotension was defined as a reduction in systolic blood pressure to $<90 \mathrm{mmHg}$ or by $>20 \%$ from the baseline combined with decrease in diastolic blood pressure to less than $60 \mathrm{mmHg}$ or by more than $20 \%$ from the baseline.

Hypotension was cured immediately by increasing the rate of IV Ringer's solution and by $5 \mathrm{mg}$ Ephedrine, intravenously. Systolic blood pressure and diastolic blood pressure were checked after 2 minute and supplement of $5 \mathrm{mg}$ Ephedrine was given until systolic blood pressure was established at 90 $\mathrm{mmHg}$ and diastolic blood pressure was established at 60 $\mathrm{mmHg}$.

Immediately after delivery of the baby, for the two groups, an arterial blood sample was drawn from a double-clamped section of an umbilical cord, collected in heparinized syringe, taken to the laboratory within one hour of collection and analyzed for blood gases and $\mathrm{pH}$, as a measure to diagnose the neonatal acidosis. Apgar scores were additionally assessed at the 1 st and the 5 th minutes.

\subsection{Statistical analysis}

All statistical analyses were done using SPSS for windows version 20.0. All continuous data were normally distributed and were expressed in mean \pm standard deviation (SD) for parametric data. Categorical data were expressed in number and percentage. The comparisons were determined using independent sample $t$-test for variables with continuous data. Chi-square test was used for comparison of variables with 
categorical data. The threshold of significance is fixed at $5 \%$ level ( $p$-value). The results were considered significant when the probability of error is less than $5 \%(p<.05)$, nonsignificant when the probability of error is more than $5 \%(p$ $>.05)$, highly significant when the probability of error is less than $0.1 \%(p<.001)$, and the smaller the $\mathrm{p}$-value obtained, the more significant are the results.

\section{RESULTS}

Table 1 presents sociodemographic characteristics of leg wrapping and control groups. The mean age of both groups was almost identical (26.5 vs 27.2 respectively). The higher percentages of leg wrapping and control groups had a secondary educational level, were housewives, and from the rural residence.

Table 1. Frequency distribution of leg wrapping and control groups according to their sociodemographic characteristics

\begin{tabular}{|c|c|c|c|c|c|c|}
\hline \multirow{3}{*}{ Characteristics } & \multirow{2}{*}{\multicolumn{2}{|c|}{$\begin{array}{l}\text { Leg wrapping group } \\
(n=44)\end{array}$}} & \multirow{2}{*}{\multicolumn{2}{|c|}{$\begin{array}{l}\text { Control group } \\
(\mathrm{n}=44)\end{array}$}} & \multicolumn{2}{|c|}{ Chi-square test } \\
\hline & & & & & \multirow{2}{*}{$\chi^{2}$} & \multirow{2}{*}{$p$} \\
\hline & $\mathbf{n}$ & $\%$ & $\mathbf{n}$ & $\%$ & & \\
\hline \multicolumn{7}{|l|}{ Age (years) } \\
\hline $19-23$ & 15 & 34.1 & 12 & 27.3 & & \\
\hline $24-29$ & 18 & 40.9 & 14 & 31.8 & & \\
\hline $30-35$ & 11 & 25.0 & 18 & 40.9 & 2.523 & .283 \\
\hline Mean \pm SD & \multicolumn{2}{|c|}{$26.5 \pm 4.7$} & \multicolumn{2}{|c|}{$27.2 \pm 5.3$} & $0.623 *$ & .535 \\
\hline \multicolumn{7}{|l|}{ Educational level } \\
\hline Basic and less & 9 & 20.5 & 3 & 6.8 & & \\
\hline Secondary & 25 & 56.8 & 33 & 75.0 & & \\
\hline University & 10 & 22.7 & 8 & 18.2 & 4.326 & .115 \\
\hline \multicolumn{7}{|l|}{ Occupation } \\
\hline Housewife & 37 & 84.1 & 40 & 90.9 & & \\
\hline Employed & 7 & 15.9 & 4 & 9.1 & 0.935 & .334 \\
\hline \multicolumn{7}{|l|}{ Residence } \\
\hline Rural & 30 & 68.2 & 25 & 56.8 & & \\
\hline Urban & 14 & 31.8 & 19 & 34.2 & 1.212 & .271 \\
\hline
\end{tabular}

*t value, Student's t-test

Table 2 clarifies baseline heart rate, systolic blood pressure, was no statistically significant difference between the two diastolic blood pressure and mean arterial pressure between groups concerning different baseline hemodynamic values. leg wrapping and control groups. It was observed that there

Table 2. Mean of the baseline heart rate, systolic blood pressure, diastolic blood pressure and mean arterial pressure between leg wrapping and control groups

\begin{tabular}{|c|c|c|c|c|}
\hline \multirow{3}{*}{ Items } & \multirow{3}{*}{$\begin{array}{l}\text { Leg wrapping group } \\
(\mathrm{n}=44) \\
\text { Mean } \pm \text { SD }\end{array}$} & \multirow{3}{*}{$\begin{array}{l}\text { Control group } \\
(n=44) \\
\text { Mean } \pm \text { SD }\end{array}$} & \multicolumn{2}{|c|}{ Student's $t$-test } \\
\hline & & & \multirow{2}{*}{$t$} & \multirow{2}{*}{$p$} \\
\hline & & & & \\
\hline Heart Rate (bpm) & $103.4 \pm 11.8$ & $107.8 \pm 13.6$ & 1.616 & .110 \\
\hline Systolic blood pressure (mmHg) & $127.0 \pm 14.0$ & $126.7 \pm 16.8$ & 0.110 & .912 \\
\hline Diastolic blood pressure (mmHg) & $77.1 \pm 11.0$ & $78.5 \pm 16.1$ & 0.448 & .655 \\
\hline Mean arterial pressure (mmHg) & $93.7 \pm 11.2$ & $93.8 \pm 11.4$ & 0.019 & .985 \\
\hline
\end{tabular}

Table 3 reveals the mean of the heart rate changes after anesthesia administration between leg wrapping and control groups. There are significant heart rate changes between the two groups at $10 \mathrm{~min}$ with highly significant at $55 \mathrm{~min}$ after spinal anesthesia ( $p=.042 \& p<.001$ respectively). 
Table 3. Mean of the heart rate changes after anesthesia administration between leg wrapping and control groups

\begin{tabular}{lllll}
\hline \multirow{2}{*}{ Items } & Leg wrapping group & Control group & \multicolumn{2}{l}{ Student's $\boldsymbol{t}$-test } \\
\cline { 2 - 4 } & $\mathbf{( n = 4 4 )}$ & $\mathbf{( n = 4 4 )}$ & $\boldsymbol{t}$ & $\boldsymbol{p}$ \\
\cline { 2 - 4 } & Mean \pm SD & Mean \pm SD & 0.491 & .625 \\
\hline $5 \mathrm{~min}$ & $109.9 \pm 29.1$ & $106.7 \pm 31.3$ & 2.069 & .042 \\
$10 \mathrm{~min}$ & $98.3 \pm 17.3$ & $106.7 \pm 21.0$ & 0.142 & .887 \\
$15 \mathrm{~min}$ & $103.6 \pm 16.5$ & $104.1 \pm 16.6$ & 0.086 & .931 \\
$20 \mathrm{~min}$ & $105.0 \pm 16.5$ & $105.3 \pm 12.9$ & 0 & 1.000 \\
$25 \mathrm{~min}$ & $102.1 \pm 16.3$ & $102.1 \pm 12.9$ & 1.292 & .200 \\
$30 \mathrm{~min}$ & $98.9 \pm 25.8$ & $104.5 \pm 12.3$ & 1.422 & .159 \\
$35 \mathrm{~min}$ & $101.5 \pm 13.9$ & $105.5 \pm 12.6$ & 0.902 & .370 \\
$40 \mathrm{~min}$ & $101.6 \pm 12.5$ & $99.1 \pm 12.1$ & 0.046 & .963 \\
$45 \mathrm{~min}$ & $98.4 \pm 11.7$ & $98.6 \pm 13.3$ & 0.021 & .983 \\
$50 \mathrm{~min}$ & $92.9 \pm 9.7$ & $92.8 \pm 15.7$ & 9.863 & $<.001$ \\
\hline $5 \mathrm{~min}$ & $78.5 \pm 4.0$ & $109.0 \pm 1.4$ & & \\
\hline
\end{tabular}

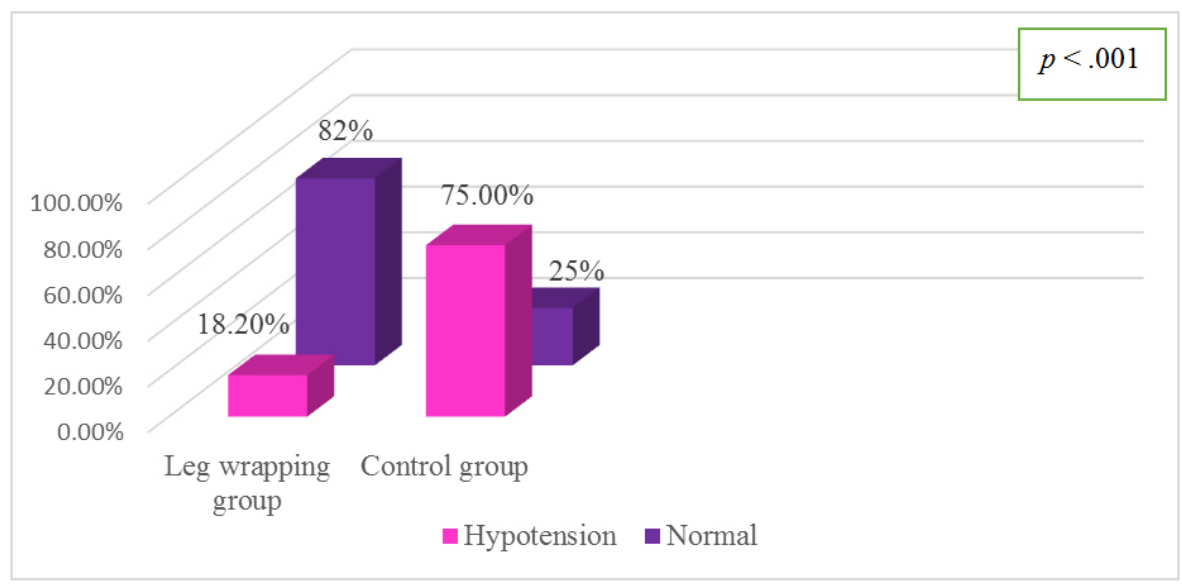

Figure 3. The incidence of hypotension in leg wrapping and control groups

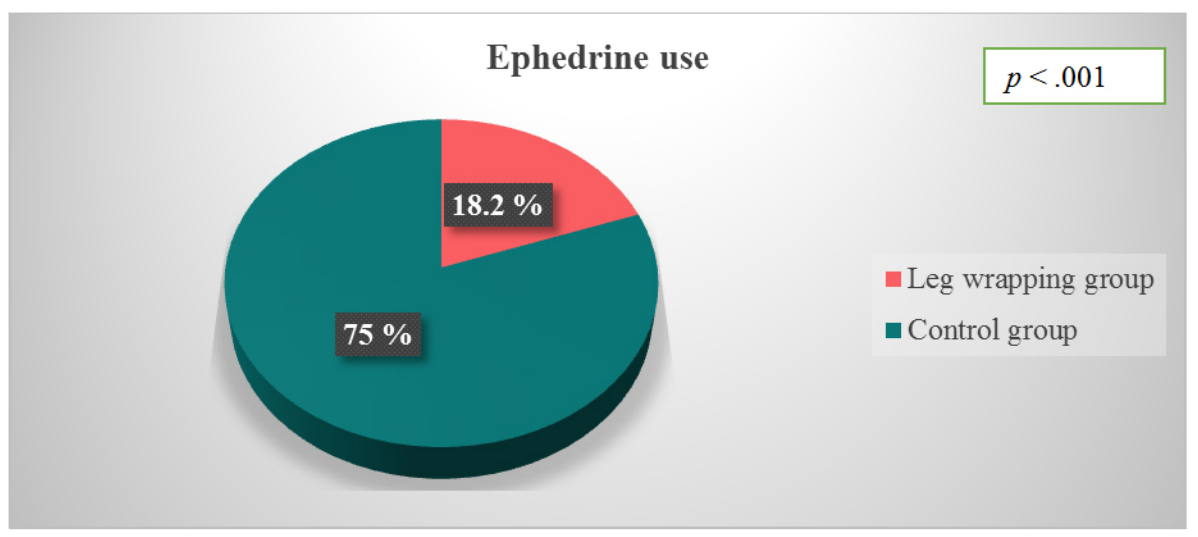

Figure 4. Comparison of Ephedrine use between leg wrapping and control groups 


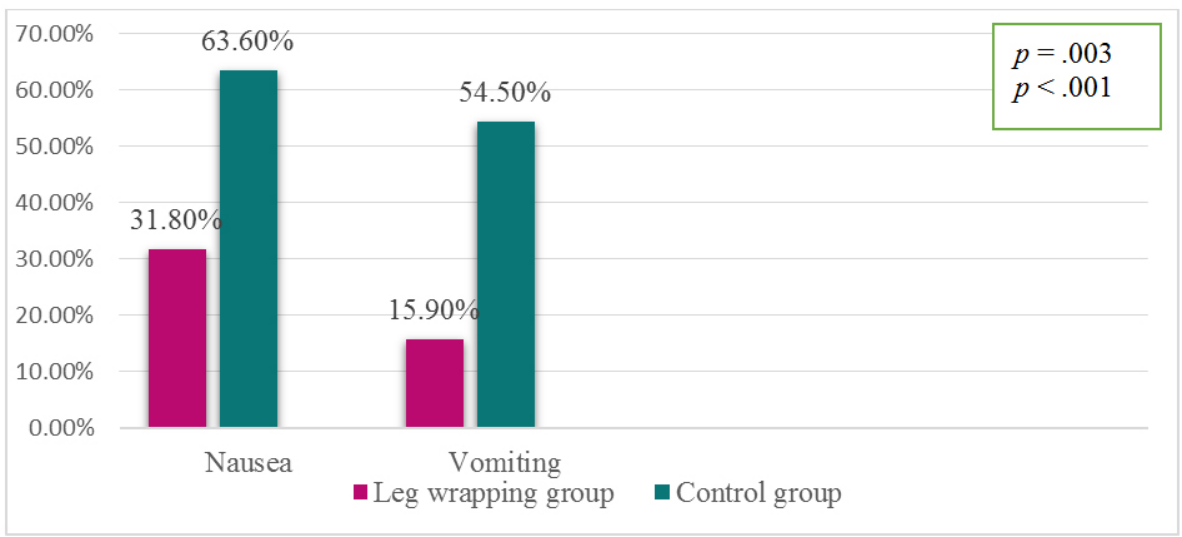

Figure 5. Comparison of signs of hypotension between leg wrapping and control groups

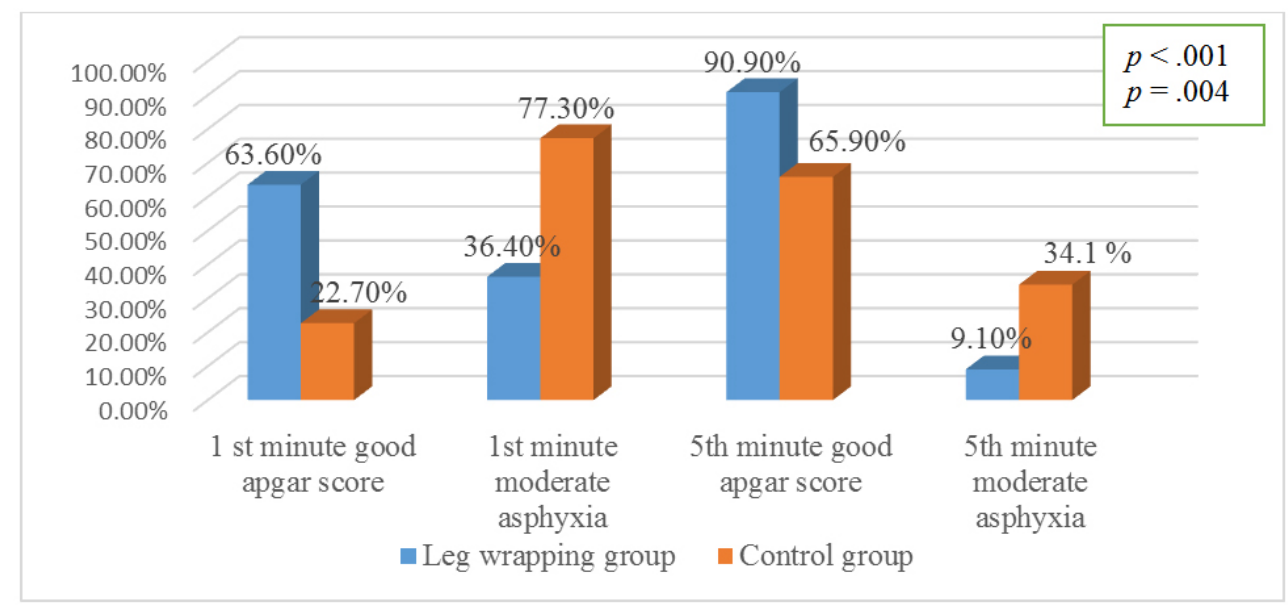

Figure 6. Comparison of the neonatal Apgar score 1st and the 5th minute between leg wrapping and control groups

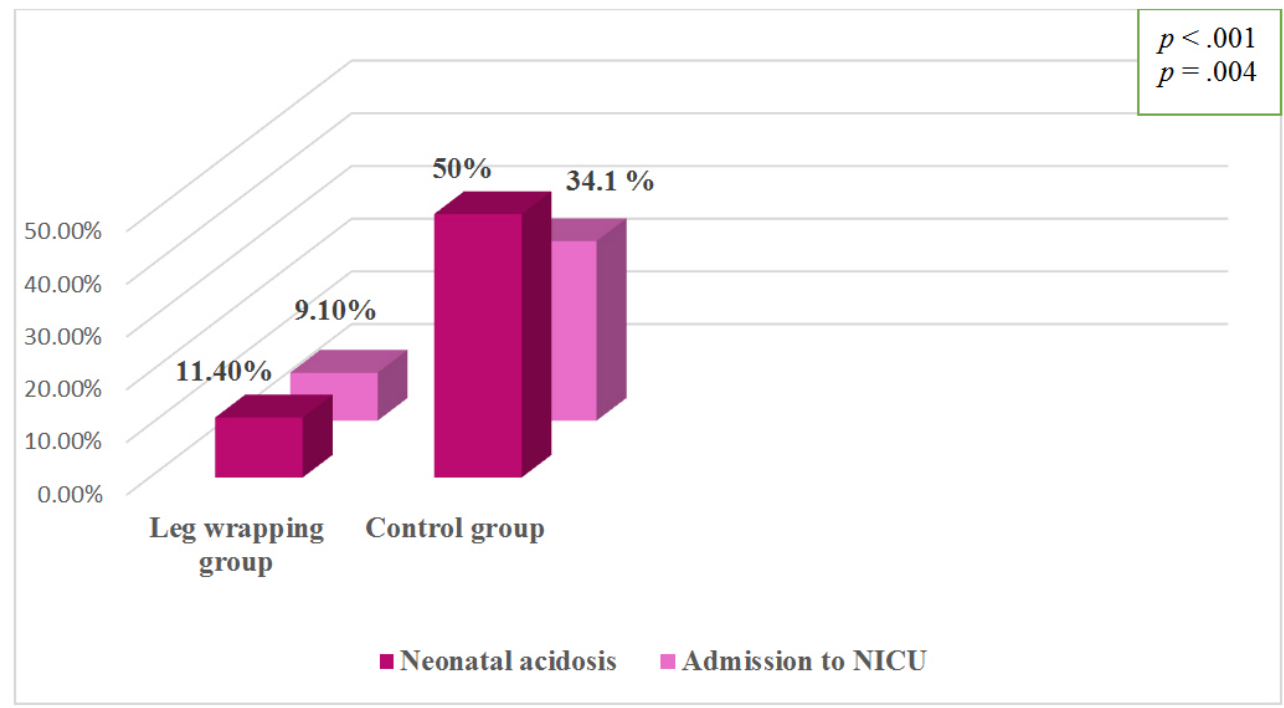

Figure 7. Comparison of the neonatal condition between leg wrapping and control groups 
Table 4. Barriers versus positive factors that affect the application of leg wrapping technique for prevention of SIH during CS from researcher's point of view

\begin{tabular}{|c|c|}
\hline Barriers & Positive factors \\
\hline Lack of support of the Directorate of Health Affairs to conduct the research & Anesthesiologist support \\
\hline Lack of cooperation of resident obstetricians & Obstetrician support \\
\hline $\begin{array}{l}\text { Lack of health care awareness regarding leg wrapping technique for } \\
\text { prevention of SIH during CS }\end{array}$ & $\begin{array}{l}\text { Availability of equipment and tools for conducting } \\
\text { the study by the researcher }\end{array}$ \\
\hline Lack of support of hospital administration & \multirow[b]{2}{*}{$\begin{array}{l}\text { Providing the necessary information regarding leg } \\
\text { wrapping technique for prevention of SIH during CS } \\
\text { to pregnant women who participated in the study }\end{array}$} \\
\hline $\begin{array}{l}\text { Medicalization system } \\
\text { Busy nurses due to lack of nursing staff and work overload } \\
\text { The absence of the culture of EBP in hospital }\end{array}$ & \\
\hline
\end{tabular}

Table 5. Barriers versus positive factors that affect the application of leg wrapping technique for prevention of SIH during CS from operating room staff's point of views

\begin{tabular}{ll}
\hline Barriers & Positive factors \\
\hline $\begin{array}{l}\text { Inability to sterilize woman's thigh in the presence of bandage at the level of } \\
\text { mid-thigh }\end{array}$ & $\begin{array}{l}\text { Performing leg wrapping technique by the } \\
\text { researcher did not depend on nurses } \\
\text { Left uterine displacement can't be performed as it leads to the drainage of liquor } \\
\text { and blood on scrub nurse and assistant obstetrician } \\
\begin{array}{l}\text { Lack of time to perform the leg wrapping technique } \\
\text { ceg wrapping technique is not included in hospital policies and regulations } \\
\text { Hospital capabilities and lack of resources }\end{array}\end{array}$ \\
\hline
\end{tabular}

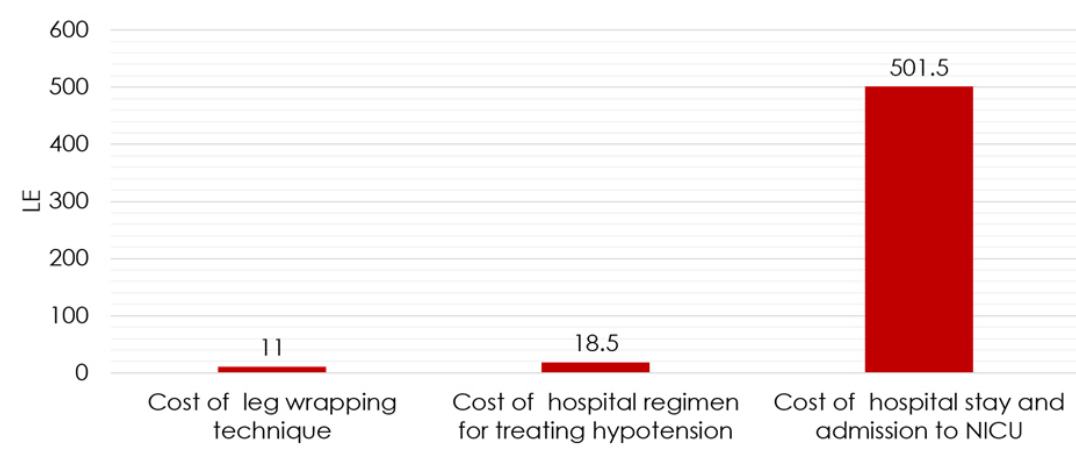

Figure 8. Comparison of the cost of the leg wrapping technique against the cost of hospital regimen for treating hypotension and admission to NICU

\section{Discussion}

The Study aimed to apply leg wrapping technique for prevention of SIH during CS. The Study aim was supported by the study findings. There was a highly statistically significant difference between leg wrapping and the control group concerning occurrences of spinal-induced hypotension and fetal acidosis. Therefore, the study hypotheses were supported.

In the current study, concerning heart rate changes after anesthesia administration between leg wrapping and control group, the result findings indicated that there were inconsistent heart rate changes, some women had an increase in heart rate with the onset of hypotension while others a decrease.

Published by Sciedu Press
Such findings are supported by Das \& Swain ${ }^{[14]}$ who reported significant increase in heart rate in control group compared to study group $(p<.05)$ during intraoperative monitoring from 4 min to 15 minutes with highly significant from 6th minute to 10 th minute $(p<.001)$. Moreover the results were in agreement with Elgzar \& Ebrahim ${ }^{[16]}$ who stated in their study conducted in Egypt that there was a statistically significant difference in the mean heart rate between intervention group and control group $(p<.05)$ throughout the repeated measures except at 35, 45 and $50 \mathrm{~min}$, indicating lower heart rate in intervention group which correlates with the current study. 
Contradictory results reported by Kuhn et al. ${ }^{[8]}$ results who stated that cardiac output and heart rate were significantly lower among phenylephrine group compared with leg wrapped group in Norwegian pregnant women. The difference between the present study finding and such finding may be due to the pharmacologic effect of phenylephrine which produces systemic vasopressor effect and subsequently decreases heart rate. Leg wrapping is nursing procedure and is difficult to be compared with medications, which have systemic effect. Also, Bagle et al. ${ }^{[17]}$ who reported no significant changes between study and control groups regarding heart rate for Indian pregnant women. They explained that some heart rate variation noted after vasopressors administration.

The current study results confirm that the decrease in the incidence of spinal-induced hypotension achieved by increasing central blood volume by elevation \& wrapping the legs and the utilization of left lateral tilt of $15^{\circ}$. Left uterine displacement of the gravid uterus appears to be a reasonable method to manage hypotension because it relieves aortocaval compression by dislocating the gravid uterus. Utilizing these three approaches together in our study decreased the spinal-induced hypotension and, as a result, there was a great reduction in the use of vasopressor by nearly $82 \%$.

The positive effect of wrapping of lower limbs as a prophylactic technique to avoid SIH has been well documented previously. Mohamed et al.; ${ }^{[12]}$ Das \& Swain.; ${ }^{[14]}$ Elgzar \& Ebrahim; ${ }^{[16]}$ Bagle et al. ${ }^{[17]}$ who found that wrapping of lower limbs just before spinal anesthesia is an effective technique to decrease frequency of severe hypotension episodes and dose requirement of vasopressor used, a difference which was significant, both clinically and statistically.

Moreover, Hasanin et al. ${ }^{[18]}$ concluded that the changing position of Egyptian parturients after the subarachnoid block from supine to left lateral tilted position leading to increased cardiac output and mean arterial pressure. There is no difference between both tilt angles (15 degrees and 30 degrees). Additionally, İnce et al. ${ }^{[19]}$ supported the present study findings in their study in Turkey in which they found that the hypotension incidence and ephedrine consumption in the passive leg raising group was significantly decreased when compared with the control group $(p=.02, p<.01$ respectively).

Contradictory results reported by Kundra et al. ${ }^{[20]}$ who reported that manual uterine displacement efficiently decreased both the incidence of hypotension and the vasopressors administration compared with 15 degrees left lateral tilt of the table $(4.4 \%$ v.s. $40 \% ; p<.001)$.
Also, Jabalameli et al. ${ }^{[21]}$ who found that $51.3 \%$ of all cases who received bandage experienced hypotension. However, those who received ephedrine plus bandage experienced better maternal outcomes compared with those who received crystalloid or colloid plus bandage. This might be related to the fact that wrapping was done after the spinal injection, while we wrapped the legs just before.

Regarding signs of hypotension, the current study found that nausea and vomiting occurred in the control group more than those in the leg wrapping group. These results may be due to that, incidence of hypotension was high among the control group, which may cause brainstem ischemia, which is supposed to activate the circulatory, respiratory, and vomiting centers gathered together in the medulla.

Such findings are supported by Mohamed et al.;[?] Das \& Swain.; ${ }^{[14]}$ Elgzar \& Ebrahim; $;{ }^{[16]}$ Putri et al. ${ }^{[22]}$ who stated that the incidence of nausea and vomiting after spinal anesthesia has remained high in the control group compared to study group and has a significant adverse effect on women satisfaction about the overall delivery experience.

This study finding was in disagreement with Sujata et al. ${ }^{[23]}$ who stated that two Indian women in the control group and three in the mechanical pump group had nausea, but none vomited ( $p$ value $=.68$ ). Additionally, contrary to the current study finding, Zasa et al. ${ }^{[13]}$ who reported that incidence of nausea and/or vomiting was not statistically different between both groups $(p=.228)$.

With respect to the neonatal condition, as assessed by Apgar scores and umbilical cord blood gases, the current study revealed that, neonates were had higher Apgar scores at 1st and 5th minute in leg wrapping group, and there was a significant difference between the two groups $(p<.001, p=.004$ respectively). Neonatal acidosis occurred in 5 of 44 neonates in leg wrapping group compared to half of the neonates in the control group. 4 neonates in leg wrapping group admitted to NICU while more than one-third of neonates admitted to NICU in the control group. There was a significant difference between both groups $(p<.001, p=.004$ respectively).

These results may be due to that uteroplacental circulation and therefore fetal wellbeing depends on maternal blood pressure. So, maternal hypotension has a strong correlation with neonatal academia. Consequently, if SBP, DBP, and MAP are normal in the intervention group in the present study, then the incidence of neonatal acidosis is anticipated to be less in the study group. Moreover, the ephedrine administration was more common in the control group. American guidelines from 2016 reported that ephedrine might lead to more fetal acidosis during CS under spinal anesthesia. 
Such findings are supported by Mohamed et al.; Elgzar \& Ebrahim $^{[12,16]}$ who reported that there was a statistically significant difference between both groups concerning the Apgar score of the neonate at the 1st minute and the neonatal acidosis.

In contrast to this study; Lee et al. ${ }^{[24]}$ who identified in their study carried out in U.S.A that there were no significant differences between groups in umbilical artery $\mathrm{pH}$ or Apgar scores. Also, Peyronneta et al. ${ }^{[25]}$ who reported that no Apgar scores were below 7 at 5 th min and there were no significant differences among the groups in Apgar scores at the 5th minute $(p=.43)$. Umbilical cord blood $\mathrm{pH}$ was not below 7.2 in any case and no significant difference was detected among the groups regarding fetal acidosis $(p=.55)$ also, no significant difference was noted among the groups regarding NICU admission $(p=.71)$.

To the best of our knowledge, this is the first study that explored the feasibility of application of leg wrapping technique for avoidance of SIH during CS. According to researcher's point of view regarding major barriers that hinder the application of leg wrapping technique were lack of support of the Directorate of Health Affairs which is related to Ministry of Health and Population to conduct the research, lack of cooperation of resident obstetricians, lack of health care awareness regarding leg wrapping technique for prevention of SIH during CS, lack of support of hospital administration, medicalization system, busy nurses due to lack of nursing staff and work overload and the absence of the culture of EBP in hospital.

Regarding major positive factors that facilitate the application of leg wrapping technique for prevention of SIH during CS from researcher's point of view were anesthesiologist support, obstetrician support, availability of equipment and tools for conducting the study by the researcher and providing necessary information regarding leg wrapping technique for prevention of SIH during CS to pregnant women who participated in the study.

According to operating room staffs' points of view regarding major barriers that hinder the application of leg wrapping technique for prevention of SIH during CS, all the operating room staffs reported inability to sterilize woman's thigh in the presence of bandage at the level of mid-thigh, left uterine displacement can't be performed as it leads to the drainage of liquor and blood on scrub nurse and assistant obstetrician, lack of time to perform the leg wrapping technique, leg wrapping technique is not included in hospital policies and regulations, hospital capabilities and lack of resources.

The results of the current study revealed that performing

Published by Sciedu Press leg wrapping technique by the researcher didn't depend on nurses, availability of equipment and tools for conducting the study by the researcher and researcher has good communication skills and personal rapport were the positive factors that facilitate the application of leg wrapping technique for prevention of SIH during CS according to operating room staffs' points of view.

Regarding the cost of the leg wrapping technique against the cost of hospital regimen for treating hypotension and admission to (NICU), the current study revealed that the leg wrapping technique was cost effective compared to the cost of hospital regimen for treating hypotension and admission to (NICU) (11 EGP, 18.5 EGP, and 501.5 EGP respectively).

Leg wrapping technique constitutes an attractive technique to avoid spinal-induced hypotension as it is an easy, cost effective, non-pharmacological technique and well accepted by women. In the current study, no adverse effects were observed and the women were satisfied with the leg wrapping technique.

\section{Limitation of the study}

Hemodynamic assessment of participants was based only on heart rate and blood pressure. We suppose that the utilization of advanced cardiac output monitors in future studies might be more informatory for the exact effect of leg wrapping on maternal hemodynamics, as cardiac output is a better indicator of uteroplacental perfusion than upper arm blood pressure measurement. The left lateral tilt of 15 degrees could not be preserved during the study as some obstetricians found this position unacceptable and some women felt as if they were falling.

\section{Conclusion}

Based on the findings of the current study, it is concluded that, the wrapping of lower limbs with simple elastic crepe bandage just before spinal anesthesia is an effective method to decrease the incidence of SIH to a significant degree during cesarean section with a consequent decrease in the use of strong vasopressor therapy and their unnecessary pharmacological action. Also, it reduced the incidence of neonatal acidosis. Economically, leg wrapping technique was cost effective compared to the cost of the hospital regimen for treating hypotension and admission to (NICU).

\section{Recommendations}

Based on the current study findings, the following can be recommended:

- The leg wrapping during CS should be included in the maternal hospitals' protocol for preventing SIH. 
- The leg wrapping technique should be integrated with the context of the health care system in maternity hospitals.

- Continuous training programs for health care providers in the maternal hospitals regarding the application of non-pharmacological preventive measures for spinalinduced hypotension during cesarean delivery especially leg wrapping technique.

- Including educational program about how to apply preventive measures for spinal-induced hypotension during cesarean delivery $\&$ health benefits of nonpharmacological methods and vasopressor risks into maternity nursing undergraduate curriculum.

- Further researches includes replicating the current study on a larger population and different setting and evaluating the effect of other techniques of lower leg compression during cesarean section by using advanced cardiac output monitors.

\section{ACKNOWLEDGEMENTS}

We would like to thank all participants for their cooperation during the study. We also acknowledge the help and patience of the staff in the operating unit at Mansoura General Hospital.

\section{CONFLicts OF InTEREST Disclosure}

The authors declare that there is no conflict of interest statement.

\section{REFERENCES}

[1] WHO. World Health statistics 2012. 2012. Available from: http://www. who.int/gho/publications/world_health_ statistics/2018/en/

[2] Ministry of Health and Populations [Egypt], El-Zanaty Associates [Egypt], ICF International. The 2014 Egypt Demographic and Health Survey (2014 EDHS). Main Findings. Cairo, Egypt. 2015; 1-52.

[3] Chooi C, Cox JJ, Lumb RS, et al. Techniques for preventing hypotension during spinal anesthesia for cesarean section. Cochrane Database of Systematic Reviews. 2017.

[4] Shisanya MS, Morema EN. Determinants of Maternal Satisfaction with Spinal Anaesthesia Care for Caesarian Delivery at the Kisumu County Hospital. IOSR Journal of Nursing and Health Science. 2017. https://doi.org/10.9790/1959-0601089195

[5] Singh TH, Thokchom RS, Sinam M, et al. Prophylactic intravenous ephedrine for the prevention of hypotension in the cesarean section during spinal anesthesia: A comparative study. Journal of Medical Society. 2016; 30(2): 116. https://doi .org/10.4103/0972-4 958.182922

[6] Butwick AJ, Columb MO, Carvalho B. Preventing Spinal Hypotension During Cesarean Delivery: What is the Latest? Obstetric Anesthesia Digest. 2015; 35(4): 176-177. https://doi.org/10.109 7/01.aoa.0000472691.37136.22

[7] Gibbs MW, van Dyk D, Dyer RA. Managing spinal hypotension during caesarean section: An update. South African Medical Journal. 2018; 108(6): 460-463. https://doi.org/10.7196/SAMJ . 2018 v108i6. 13373

[8] Kuhn JC, Hauge TH, Rosseland LA, et al. Hemodynamics of phenylephrine infusion versus lower extremity compression during spinal anesthesia for cesarean delivery: a randomized, doubleblind, placebo-controlled study. Anesthesia \& Analgesia. 2016; 122(4): 1120-1129. PMid:26991619 https://doi.org/10.121 3/ANE. 0000000000001174

[9] Khedr NF. Preventive measures to reduce post-spinal anesthesia hypotension for elective cesarean delivery. Journal of American Science. 2011; 7: 634-640.

[10] Mercier FJ, Diemunsch P, Ducloy-Bouthors AS, et al. 6\% Hydroxyethyl starch (130/0.4) vs Ringer's lactate preloading before spinal anaesthesia for Caesarean delivery: the randomized, double-blind, multicentre CAESAR trial. British Journal of Anaesthesia. 2014;
113(3): 459-467. PMid:24970272 https://doi.org/10.1093/ bja/aeu103

[11] Xu S, Zhao Q, Wang F, et al. Crystalloid versus Colloid in Stabilizing Hemodynamic of Patients Undergoing Cesarean Section with Neuraxial Anesthesia. SCIENCE INSIGHTS. 2019; e0012.

[12] Mohamed AI, Elazhary RAH, Abdelhady RM, et al. Utilization of lower leg compression technique for reducing spinal induced hypotension, and related risks for mothers and neonates during cesarean delivery. Journal of Nursing Education and Practice. 2016; 6(7): 11 https://doi.org/10.5430/jnep.v6n7p11

[13] Zasa M, Conci E, Marchignoli A, et al. Comparison of two different approaches to hypotension following spinal anaesthesia for Caesarean delivery: effects on neonatal and maternal wellbeing. Acta Bio Medica Atenei Parmensis. 2015; 86(1): 45-52.

[14] Das P, Swain S. Effect of leg wrapping on hemodynamics and associated complications in caesarean section: a randomized prospective study. International Journal of Research in Medical Sciences. 2016; 4(10): 4408-4415. https://doi.org/10.18203/2320-6012.ij rms20163302

[15] Naghibi K, Rahimi M, Mashayekhi Z. A Comparison of Intravenous Ephedrine or Phenylephrine, for Prevention of Post spinal Hypotension during Elective Lower Abdominal Surgery: A Randomized, Double-blind Case-control Study. Advanced Biomedical Research. 2017.

[16] Elgzar WTE, Ebrahim HA. Effect of lower leg compression during cesarean section on post-spinal hypotension and neonatal hemodynamic parameters: nonrandomized controlled clinical trial. International Journal of Nursing Sciences. 2019

[17] Bagle AA, Vishnu A, Kumar A, et al. Evaluation of leg wrapping for the prevention of post spinal hypotension in cesarean section under spinal anesthesia. Anesthesia, Essays and Researches. 2017; 11(2): 439. PMid:28663637 https ://doi .org/10.4103/0259-1 162.194564

[18] Hasanin A, Soryal R, Kaddah T, et al. Hemodynamic effects of lateral tilt before and after spinal anesthesia during cesarean delivery: an observational study. BMC Anesthesiology. 2018; 18(1): 8 . PMid:29334907 https://doi.org/10.1186/s12871-018-047 3-0 
[19] İnce EM, Sizlan A, Senkal S, et al. Spinal Anestezi İle Sezaryen Yapılacak Gebelerde Pasif Bacak Kaldırma Uygulamasının Hipotansiyonu Önlemedeki Etkinliği. JARSS. 2019; 27(2): 36-44.

[20] Kundra P, Khanna S, Habeebullah S, et al. Manual displacement of the uterus during Caesarean section. Anaesthesia. 2007; 62(5): 460465. PMid:17448057 https://doi.org/10.1111/j.1365-204 $4.2007 .05025 . \mathrm{x}$

[21] Jabalameli M, Soltani HA, Hashemi J, et al. A randomized comparative trial of combinational methods for preventing post-spinal hypotension at elective cesarean delivery. Journal of research in medical sciences: the official journal of Isfahan University of Medical Sciences. 2011; 16(9): 1129.

[22] Putri YS, Fuadi I, Bisri T. Efek Penggunaan Leg Wrapping terhadap Kejadian Hipotensi Selama Anestesi Spinal pada Pasien Seksio Sesarea. Journal Anestesi Perioperatif. 2016; 4(3): 191-197. https://doi.org/10.15851/jap.v4n3.903
[23] Sujata N, Arora D, Panigrahi BP, et al. A sequential compression mechanical pump to prevent hypotension during elective cesarean section under spinal anesthesia. International Journal of $\mathrm{Ob}$ stetric Anesthesia. 2012; 21(2): 140-145. PMid:22398360 https : //doi.org/10.1016/j.ijoa.2012.01.003

[24] Lee AJ, Landau R, Mattingly JL, et al. Left Lateral Table Tilt for Elective Cesarean Delivery under Spinal Anesthesia Has No Effect on Neonatal Acid-Base Status A Randomized Controlled Trial? Anesthesiology: The Journal of the American Society of Anesthesiologists. 2017; 127(2): 241-249. PMid:28598894 https : //doi.org/10.1097/ALN.0000000000001737

[25] Peyronnet V, Roses A, Girault A, et al. Lower limbs venous compression reduces the incidence of maternal hypotension following epidural analgesia during term labor. European Journal of Obstetrics \& Gynecology and Reproductive Biology. 2017; 219: 94 99. PMid:29073508 https : //doi .org/10.1016/j. ejogrb. 201 7.10 .016 